\title{
Dealing with COVID-19 in a Pediatric Rheumatology Unit in Italy
}

\author{
Stefania Costi ${ }^{1} \cdot$ Roberto Caporali $^{2,3} \cdot$ Rolando Cimaz $^{2,3}$
}

Published online: 16 April 2020

(c) Springer Nature Switzerland AG 2020

Italy has been the first nation in the European Union affected by the Coronavirus epidemic and is the first in the world for number of reported deaths. As of March 31, 101,739 positive cases were reported $(42,161$ of them in the Lombardy region), as well as 11,591 deaths. With regard to children, we have 1226 positive cases between the ages of 0 and 18 years, that is, $1.3 \%$ of total infections. Based on available evidence, children do not appear to be at higher risk for COVID-19 than adults; indeed, they appear to experience a milder form of the infection. The reasons for the relative resistance of children are still unknown, and may be related to a different immune response, to healthier respiratory tracts secondary to shorter air pollution exposure and absence of cigarette smoke, and to a lack of comorbidities [1].

In our Pediatric Rheumatology Unit, we treat more than 2000 young patients with chronic rheumatological inflammatory disorders, and almost 500 of them are on biologic therapies. Parents are very worried about the possibility of their children contracting COVID-19 infection due to immunosuppression. We receive hundreds of telephone calls, and feel that a uniform response by healthcare providers (and pediatric rheumatologists in particular) is necessary in order to avoid wrong messages and possibly a negative outcome on patients' health due to incorrect treatment recommendations.

In our center, we recommend simple measures to prevent contact with infected subjects and spread of the virus. In particular, we underline the importance of regular cleaning of hands with soap or alcohol-based sanitizers, of avoiding contact with people who manifest respiratory symptoms, of coughing or sneezing not on palms but into the elbow, and of not touching their own face as much as possible. We $d o$

Stefania Costi

stefania.costi@unimi.it

Università degli Studi di Milano, Milan, Italy

2 ASST G.Pini-CTO, Milan, Italy

3 Department of Clinical Sciences and Community Health and Research Center for Adult and Pediatric Rheumatic Diseases, Università degli Studi di Milano, Milan, Italy not recommend emergency department visits unless strictly necessary, but if new symptoms arise a national telephone number is available, and we have also instituted in our hospital an on-call specific number to answer clinical questions or doubts about behaviors to adopt. Outpatient visits and elective hospitalizations are limited to those considered nondeferrable. Currently, recommendations of rheumatologic societies both in Italy and Europe suggest continuation of all immunosuppressant therapies as usual. In fact, withdrawal of medications may cause a flare of inflammatory disease, which can lead to higher infection risk [2]. In case of fever or respiratory symptoms, patients should consult the treating physician. Following these rules, up to the time of writing (31 March 2020), we have not yet heard of a COVID-19 case in our patient cohort, and disease relapses have been mild and no different from before the epidemic spread.

In recent days, scientific societies such as the European League Against Rheumatism (EULAR) and the Italian Society of Rheumatology (SIR) have created databases to monitor and report outcomes of COVID-19 in patients suffering from autoimmune or autoinflammatory diseases $[3,4]$. The aim is to understand how epidemiological factors such as age, comorbidities, and different immunosuppressant treatments can influence the course and outcomes of the COVID19 infection. We believe that, in this complicated moment for our healthcare system, we can contribute to increasing knowledge about a poorly understood pathogen and may help pediatric rheumatologists to make better informed therapeutic decisions.

Unfortunately, we still know too little about the role of immunosuppressant agents in relation to COVID-19 infection. A stronger immune response, typical of the adult patient, could lead to a hyperactivation of the immune system and massive cytokine release associated with the development of acute respiratory distress syndrome (ARDS) occurring in the most aggressive patterns of COVID-19 infection. In this scenario, drugs commonly used for the treatment of autoimmune diseases have been introduced for the management of critical cases of COVID-19. The ideal treatment for cytokine storm syndrome (CSS) induced by 
COVID-19 infection is still unknown, but the role of rheumatologist could be crucial. First of all we are used to managing cytokine-targeting therapies and are familiar with their side effects. In addition, rheumatologists have a strong background in understanding the immune system and can assist and work together with other specialists to treat those patients who develop CSS.

Currently, in Italy, six studies have been approved by the Italian Medicines Agency (AIFA): two of these involve the investigative antiviral drug remdesivir. The remaining trials involve drugs used in pediatric rheumatology; two are focused on tocilizumab (an anti-IL-6R monoclonal antibody approved for juvenile idiopathic arthritis [JIA]), one on sarilumab (which also targets IL-6R and is in development for JIA) and another investigating intravenous administrations of emapalumab (anti-IFN $\gamma$ ) in combination with anakinra (an IL-1 receptor antagonist, approved for cryopyrin-associated periodic syndromes and JIA). Moreover, a proposal for another open-label, phase II study to evaluate the efficacy and safety of colchicine treatment for Covid-19 infection is currently under evaluation by AIFA.

Another drug that is being investigated widely is hydroxychloroquine (HCQ), an immunomodulatory agent commonly used in autoimmune diseases such as rheumatoid arthritis and systemic lupus erythematosus. However, its effectiveness in treating COVID-19 pneumonia is still under evaluation. There are controversial opinions about its efficacy in treating or preventing COVID-19 infection and until results from large clinical trials are available, the European Medicines Agency (EMA) recommends its use only in the context of clinical trials, also to avoid consuming drug stocks. In fact, stockpiling of HCQ by otherwise healthy individuals is endangering rheumatology patients, who can no longer get hold of their regular prescription as there is little available. In some countries, prescribing of this medicine has been restricted to reduce the risk of shortages. Currently, there is only one registered completed Chinese study on HCQ, which evaluated the efficacy and safety of HCQ in this context, and the trial failed to demonstrate statistical superiority over placebo [5]. Other trials on HCQ are ongoing, both for treatment of patients with proven COVID-19 infection as well as for its prevention; there is an urgent need to understand whether immunosuppressants could be a valid treatment option, and what type of patient will benefit.

With regard to pediatric populations, treatment indications for COVID-19 are not yet well defined. Based on a Chinese consensus, management is based on respiratory support and on the use of corticosteroids and intravenous immunoglobulins in selected cases [6]. What we know about pediatric immunosuppressed patients is derived from the experience of The Hospital Papa Giovanni XXIII in Bergamo, a large pediatric hepatology and liver transplantation center. Out of about 200 transplant recipients, including ten inpatients and 100 with autoimmune liver disease, none have developed clinical pulmonary disease, despite three having tested positive for SARS-CoV-2 [7]; therefore, data suggest that immunosuppressed patients may not be at increased risk of severe infection compared with the general population. In addition, very recent Italian data show that adult patients with chronic arthritis and treated with biological DMARDs or traditional DMARDs do not seem to be at increased risk of respiratory or life-threatening complications from SARSCoV-2 compared with the general population [8].

Until results from clinical trials of antiviral and immunomodulatory drugs are available, we will obviously continue to follow daily national recommendations and keep emphasizing that simple hygiene measures and social isolation when prescribed by national authorities are the best, and for the time being, the only way to avoid epidemic spread. Protecting our young patients, as well as ourselves, is our mission now.

\section{Compliance with Ethical Standards}

Conflict of interest The authors declare that they have no conflict of interest.

Funding No sources of funding were used to support the writing of this article. This article does not contain any studies with human participants performed by any of the authors.

\section{References}

1. Lee PI, Hu YL, Chen PY, Huang YC, Hsueh PR. Are children less susceptible to COVID-19? J Microbiol Immunol Infect. 2020. https://doi.org/10.1016/j.jmii.2020.02.011.

2. Favalli EG, Ingegnoli F, De Lucia O, Cincinelli G, Cimaz R, Caporali R. COVID-19 infection and rheumatoid arthritis: Faraway, so close! Autoimmun Rev. 2020. https://doi.org/10.1016/j. autrev.2020.102523.

3. EULAR COVID-19 Rheumatological database. 2020. https:// www.redcap.rss.mhs.man.ac.uk/surveys/?s=NEXNJFWX38.

4. SIR COVID-19 database. 2020. https://www.reumatologia.it/ cmsx.asp?IDPg=1092.

5. Chen J, Liu D, Liu L, Liu P, Xu Q, Xia L, et al. A pilot study of hydroxychloroquine in treatment of patients with common coronavirus disease-19 (COVID-19). J Zhejiang Univ (Medical Science). 2020. https://doi.org/10.3785/j.issn.1008-9292.2020.03.03.

6. Shen K, Yang Y, Wang T, Zhao D, Jiang Y, et al. Diagnosis, treatment, and prevention of 2019 novel coronavirus infection in children: experts' consensus statement. World J Pediatr. 2020. https://doi.org/10.1007/s12519-020-00343-7.

7. D'Antiga L. Coronaviruses and immunosuppressed patients. The facts during the third epidemic. Liver Transpl. 2020. https://doi. org/10.1002/lt.25756.

8. Monti S, Balduzzi S, Delvino P, Bellis E, Quadrelli VS, Montecucco C. Clinical course of COVID-19 in a series of patients with chronic arthritis treated with immunosuppressive targeted therapies. Ann Rheum Dis. 2020. https://doi.org/10.1136/annrh eumdis-2020-217424. 